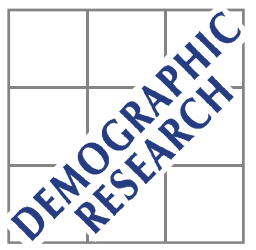

Demographic Research a free, expedited, online journal of peer-reviewed research and commentary in the population sciences published by the Max Planck Institute for Demographic Research Konrad-Zuse Str. 1, D-18057 Rostock · GERMANY www.demographic-research.org

DEMOGRAPHIC RESEARCH

VOLUME 12, ARTICLE 3, PAGES 51-76

PUBLISHED 10 MARCH 2005

www.demographic-research.org/Volumes/Vol12/3

DOI: $10.4054 /$ DemRes.2005.12.3

Research Article

\title{
Intrinsically dynamic population models
}

\section{Robert Schoen}

(C) 2005 Max-Planck-Gesellschaft. 


\section{Table of Contents}

$1 \quad$ Introduction $\quad 52$

$2 \quad$ The two age group Intrinsically Dynamic Model 53

2.1 Analyzing Leslie Matrices 53

2.2 The Constant Subordinate Eigenstructure approach 55

$3 \quad$ The three age group IDM $\quad 59$

$4 \quad$ The $n$ age group IDM $\quad 63$

$5 \quad$ IDM dynamics $\quad 64$

$5.1 \quad$ The IDM birth trajectory 64

5.2 Observed populations as IDM populations 65

$\begin{array}{lll}5.3 & \text { Transitions between stable population regimes } & 67\end{array}$

5.4 Momentum following a gradual or irregular decline $\quad 69$

to zero growth

$\begin{array}{lll}6 & \text { Summary and conclusions } & 71\end{array}$

$\begin{array}{ll}\text { References } & 72\end{array}$

$\begin{array}{ll}\text { Appendix } & 75\end{array}$ 


\title{
Intrinsically dynamic population models
}

\author{
Robert Schoen ${ }^{1}$
}

\begin{abstract}
Intrinsically dynamic models (IDMs) depict populations whose cumulative growth rate over a number of intervals is equal to the product of the long term growth rates (that is the dominant roots or dominant eigenvalues) associated with each of those intervals. Here the focus is on the birth trajectory produced by a sequence of population projection (Leslie) matrices. The elements of a Leslie matrix are represented as straightforward functions of the roots of the matrix, and new relationships are presented linking the roots of a matrix to its Net Reproduction Rate and stable mean age of childbearing. Incorporating mortality changes in the rates of reproduction yields IDMs when the subordinate roots are held constant over time. In IDMs, the birth trajectory generated by any specified sequence of Leslie matrices can be found analytically.

In the Leslie model with 15 year age groups, the constant subordinate root assumption leads to reasonable changes in the age pattern of fertility, and equations (27) and (30) provide the population size and structure that result from changing levels of net reproduction. IDMs generalize the fixed rate stable model. They can characterize any observed population, and can provide new insights into dynamic demographic behavior, including the momentum associated with gradual or irregular paths to zero growth.
\end{abstract}

\footnotetext{
${ }^{1}$ Department of Sociology, Pennsylvania State University, 211 Oswald Tower, University Park PA 16802

USA. Tel. 814-865-8869. E-mail: schoen@ pop.psu.edu
} 
Schoen: Intrinsically dynamic population models

\section{Introduction}

For nearly a century, Lotka's stable population has been the central model of mathematical demography. In a stable population, time invariant age-specific rates of birth and death produce an exponentially increasing sequence of births and an unchanging age structure (cf. Lotka 1939; Keyfitz 1968). In the 1940s, the discrete form of Lotka's continuous model was investigated, and the power of matrix theory brought to bear (Leslie 1945; Pollard 1973). Since the 1970s, the model has been extended to the multistate case, where more than one living state and the movements between them are recognized (Land and Rogers 1982; Rogers 1975; Schoen 1988). Yet the stable approach demands fixed vital rates. In a rapidly changing world characterized by numerous short term fluctuations and uncertain long term trends, a fixed rate assumption is unrealistic and often untenable.

To move beyond stable population constraints, demographers have sought to develop dynamic models, i.e. models with changing vital rates. In a pioneering work, Coale (1972) investigated patterns of dynamic rates and found approximate relationships between changing rates and the birth sequences they produced; closed form expressions eluded him. Lee (1974) considered dynamics in populations subject to external constraints. Preston and Coale (1982), building on Bennett and Horiuchi (1981), found closed form relationships that characterized any population, though they did not explicitly incorporate dynamics. Kim (1987) analyzed discrete dynamic models and found a general algebraic solution connecting changing rates and their birth sequences. However, in most instances, her solution was too complex to render in closed form. Cyclically stable populations, which arise when a fixed sequence of rates repeat indefinitely, have also been examined (Tuljapurkar 1990; Caswell 2001). An explicit solution can be found for a sequence of two rate schedules in a population with two reproductive age groups (Schoen and Kim 1994a), but most cyclical populations are far too complex for direct algebraic solution.

Recent work on "hyperstable" models has related given birth trajectories to a consistent underlying set of vital rates (Schoen and Kim 1994b; Kim and Schoen 1996) or to a sequence of fertility levels (Schoen and Kim 1997). Schoen and Jonsson (2003) presented a modified form of Quadratic Hyperstable $(\mathrm{QH})$ model that related monotonically increasing (or decreasing) fertility rates to an exponentiated quadratic birth sequence. The $\mathrm{QH}$ model provides closed form relationships that generalize the stable model, but the $\mathrm{QH}$ model is limited to one specific type of monotonic change in fertility.

The present paper extends work on dynamic modeling by introducing a new approach to modeling discrete vital rates that can relate virtually any pattern of fertility change to its resultant birth sequence. We first examine the model when there are only 2 or 3 reproductive age groups, then describe the general $n$ age group model, and finally explore model dynamics and relationships, providing numerical illustrations. 


\section{The two age group Intrinsically Dynamic Model}

\subsection{Analyzing Leslie Matrices}

To lay the foundation for the approach presented here, we begin with a brief review of the structure of population projection matrices (PPMs). A more complete treatment can be found in Caswell (2001) and the references cited there.

Consider a 2 age group population projection (Leslie) matrix that projects the beginning of the interval population to the end of the interval, i.e.

$$
A=\left(\begin{array}{cc}
a & b \\
1 & 0
\end{array}\right) .
$$

The first row elements, $a$ and $b$, indicate the contribution of the first and second age groups, respectively, at the beginning of the interval to the number of persons in the first age group at the beginning of the next interval. The first element of the second row, 1 , indicates that all persons in the first age group at the beginning of the interval survive to be in the second age group at the beginning of the next interval. Our focus here is on the birth trajectory, rather than on the age structure, and the form of $A$ generates successive numbers of births. In effect, we assume that mortality is incorporated in the "fertility" rates in the first row of $A$, and by combining mortality with fertility we simplify the structure of the Leslie matrices.

Now let the initial population be described by the vector

$$
x_{0}=\left(\begin{array}{l}
x_{10} \\
x_{20}
\end{array}\right)
$$

where $x_{j t}$ denotes the number of persons in the $j$ th age group at time $t$. We then have the projection relationship

$$
x_{1}=A x_{0}
$$

where the elements of $x_{1}$ are the "births" (i.e. numbers in the first age group) at times 1 and 0 .

Any matrix can be expressed in terms of its eigenvalues and eigenvectors (Caswell 2001). Doing so provides a mathematical decomposition of the matrix, but one with substantive interpretations in demographic usage. Accordingly, we can write

$$
A=U \Lambda V .
$$

In equation (4), $\Lambda$ is a $2 \times 2$ diagonal matrix of eigenvalues whose diagonal elements are $\lambda_{1}$ and $\lambda_{2}$. Those eigenvalues (or roots, or characteristic values) describe the growth 
rates of the two "components" implicit in matrix $A$. Eigenvalue $\lambda_{1}$, the dominant root, describes the rate of growth of the dominant (stable) component, and must be greater than $\lambda_{2}$, the growth rate of the subordinate component (cf. Keyfitz 1968). In terms of Lotka's intrinsic rate of natural increase, $r$, we have the relationship $\lambda_{1}=e^{m r}$, where $m$ is the number of years in the projection interval. (In the 2 age group case, $m=25$.) The eigenvalues are defined by the characteristic equation $|A-\lambda I|=0$, where $I$ is the identity matrix (here of order 2 ) and the vertical lines denote the determinant. With the characteristic equation of $A$ given by

$$
-\lambda(a-\lambda)-b=0
$$

we have the eigenvalues

$$
\lambda=\frac{1}{2}\left(a \pm\left(a^{2}+4 b\right)^{\frac{1}{2}}\right)
$$

where the dominant eigenvalue is provided by the positive root in equation (6).

Matrix $U$ is the matrix of right eigenvectors, and by convention is written in the form

$$
U=\left(\begin{array}{cc}
1 & 1 \\
u_{1} & u_{2}
\end{array}\right)
$$

In Leslie matrices, the right eigenvectors describe the age composition of the components. Specifically, $u_{1}$ is the number of persons in the second age group relative to a unit number in the first age group in the stable population that arises from the persistence of PPM $A$. All of the elements of the first (dominant component) right eigenvector must be real and non-negative, and it is the only right eigenvector with those properties. In the $2 \times 2$ Leslie matrix, $u_{2}$, which reflects the age structure of the subordinate component, is real but always negative. Given the form of $A$ in equation (1), we can write (Caswell 2001)

$$
u_{1}=\lambda_{1}^{-1} ; u_{2}=\lambda_{2}^{-1}
$$

where $\lambda_{2}<0$.

Matrix $V=U^{-1}$ is the matrix of left eigenvectors. Substantively, they reflect the present value of future births in each component of $A$, where those births are discounted over time by the eigenvalue of that component. The first row of $V$ relates to the first (dominant, or stable population) component. Elements in the first column give reproductive values for the first age group and those in the second column for the second age group. Given equation (1), we have

$$
V=\left(\begin{array}{cc}
1 & -\lambda_{2} \\
\frac{-\lambda_{2}}{\lambda_{1}} & \lambda_{2}
\end{array}\right)\left\{\frac{\lambda_{1}}{\left(\lambda_{1}-\lambda_{2}\right)}\right\} .
$$


The mean age of childbearing implied by matrix $A, \mu_{A}$, is given by $\left(\frac{a}{\lambda_{1}}\right)+\left(\frac{2 b}{\lambda_{1}^{2}}\right)$. In terms of roots, $\mu_{A}=\frac{\left(\lambda_{1}-\lambda_{2}\right)}{\lambda_{1}}$, the reciprocal of the scalar expression in curly brackets on the right of equation (9).

Convergence to stability occurs as $A$ is raised to higher powers (i.e. as the initial population is projected further into the future). The matrix $A^{p}$, the product of $A$ multiplied by itself $p$ times, can be seen as equal to $U \Lambda^{p} V$. Because $\lambda_{2}<\lambda_{1}, \lambda_{2}^{p}$ becomes insignificant in comparison with $\lambda_{1}^{p}$ as p becomes large. After a sufficiently long period, say $P$ intervals, $\lambda_{2}^{P}$ can be considered zero, $A^{P}$ and becomes a "rank one" matrix, i.e. becomes equal to $\lambda_{1}^{P}$ times the product of the first column of $U$ and the first row of $V$ (Caswell 2001; for a thorough discussion of the process of convergence, see Keyfitz 1968.)

\subsection{The Constant Subordinate Eigenstructure approach}

To specify an analytically useful dynamic model, we need to find an algebraic expression for the product of a sequence of Leslie matrices when those matrices are changing over time. That is a difficult problem because matrix multiplication is generally not commutative. As a result, the desired product generally cannot be written in closed form, but only as a product integral which must be evaluated numerically (cf. Gantmacher 1959).

To obtain a special case where the product of PPMs is analytically tractable, we seek an appropriate simplifying assumption. An appealing possibility is to have the growth rate over a number of intervals equal the product of the growth rates of each of those intervals. That greatly simplifies the specification of the birth sequence in terms of the fertility rates of each interval, the crucial relationship we seek to determine. Tuljapurkar (1990: 8385 ) noted that when Leslie matrices shared a common set of reproductive values, overall growth equaled the product of the $\lambda_{1}$ 's of the individual matrices. Schoen (2003) examined multistate models with uniform natural increase across model states, and found population projection matrices that had both that multiplicative property in the growth rates and constant relative reproductive values. In Leslie matrices with no mortality, like those of equation (1), equation (8) shows that an eigenvalue constant over time has an eigenvector that is constant over time. Accordingly, let us consider Leslie matrices where only the dominant eigenvalue varies over time, while the subordinate eigenvalue(s) remain constant. A sequence of such Leslie matrices should have the desired multiplicative property in the growth rates.

To begin, we make use of the algebraic relationship that exists between the roots and coefficients of a quadratic equation (Birkoff and MacLane 1959). Specifically, from equation (5), the quadratic characteristic equation $\left[\lambda^{2}-a \lambda-b=0\right]$, has $a$ as the sum of the two roots and $-b$ as the product of the two roots. Our standard but time varying PPM 
Schoen: Intrinsically dynamic population models

can thus be written as

$$
A_{t}=\left(\begin{array}{cc}
\lambda_{1 t}+\lambda_{2} & -\lambda_{1 t} \lambda_{2} \\
1 & 0
\end{array}\right)
$$

where the time $(t)$ subscript has been added to dominant root $\lambda_{1}$. Using equation (6), it can readily be verified that $a_{t}=\lambda_{1 t}+\lambda_{2}, b_{t}=-\lambda_{1 t} \lambda_{2}$ and $\lambda_{1 t}$ and $\lambda_{2}$ are the eigenvalues of $A_{t}$. Subordinate roots are not commonly encountered in demographic work, though the ratio $\frac{\lambda_{2}}{\lambda_{1}}$ is related to the rate of convergence to stability (Kim and Schoen 1993). However, equation (10) shows that the elements of our base Leslie matrix can be expressed as simple, symmetric functions of the dominant and subordinate roots. While that relationship is not new, its implications for demographic analysis have not been appreciated.

Now consider the product $M_{0,2}=A_{2} A_{1}$. In terms of its own eigenstructure, the product matrix $M_{0,2}$ can be written in the form of equation (4) as

$$
M_{0,2}=U_{M 2} \Lambda_{M 2} V_{M 2}
$$

and we find that

$$
\begin{gathered}
U_{M 2}=\left(\begin{array}{cc}
1 & 1 \\
\frac{\lambda_{11}+\lambda_{2}}{\lambda_{11}\left(\lambda_{12}+\lambda_{2}\right)} & \frac{1}{\lambda_{2}}
\end{array}\right) \\
\Lambda_{M 2}=\left(\begin{array}{cc}
\lambda_{12} \lambda_{11} & 0 \\
0 & \lambda_{2}^{2}
\end{array}\right)
\end{gathered}
$$

and

$$
V_{M 2}=\left(\begin{array}{cc}
1 & -\lambda_{2} \\
\frac{-\lambda_{2}\left(\lambda_{11}+\lambda_{2}\right)}{\lambda_{11}\left(\lambda_{12}+\lambda_{2}\right)} & \lambda_{2}
\end{array}\right)\left\{\frac{\lambda_{11}\left(\lambda_{12}+\lambda_{2}\right)}{\left.\lambda_{12} \lambda_{11}-\lambda_{2}^{2}\right)}\right\} .
$$

Paralleling equation (9), we denote the scalar factor on the right side of equation (14) as $1 / \mu_{M 2}$.

Because of the constant subordinate eigenvector restriction, that product has three important properties (Gantmacher 1959). First, the desired multiplicative property holds: the eigenvalues of $M_{0,2}$ are equal to the products of the eigenvalues of $A_{1}$ and $A_{2}$. Thus the sequence of PPMs $A_{2} A_{1}$ can be termed "intrinsically dynamic" in the sense that their product matrix $(M)$ grows at a rate that is equal to the product of their individual growth rates.

Second, the subordinate right eigenvector of all three matrices is identical. That follows from the assumption that the subordinate vector, and hence the subordinate root, is constant over time. 
Third, the relative sizes of the first row elements of the left eigenvector matrices of all three PPMs are equal. However, the scalar factor associated with $V_{M 2}$ (that can be interpreted as reflecting the reciprocal of the mean age at childbearing) does vary over time.

The dominant right eigenvector of $M$ is not constant, allowing the age composition of the dynamic model to change over time. To determine how it changes in the long term, consider the situation at time $P$, when only the dominant component of the dynamic population remains. The form of equations (11)-(14) indicates that the rank one product matrix, $M_{0, P}$, can be written in terms of its dominant root, its dominant right (column) eigenvector, and its dominant left (row) eigenvector as

$$
M_{0, P}=\prod_{j=1}^{P} \lambda_{1 j}\left(\begin{array}{c}
\frac{1}{\mu_{M P}} \\
\frac{1}{\lambda_{1 P} \mu_{M, P-1}}
\end{array}\right)\left(1-\lambda_{2}\right) .
$$

Equation (15) incorporates the scalar mean age at childbearing into the column vector, leaving the row vector constant. Let element $(i, j)$ be the element in the $i$ th row and $j$ th column of a matrix. At time $P$, the $(1,1)$ element of $M$ is the product of the $P$ dominant eigenvalues times the factor $1 / \mu_{M P}$. The $(2,1)$ element of $\mathrm{M}$ at time $P$ is the $(1,1)$ element of $\mathrm{M}$ at time $P-1$, i.e. the product of the first $(P-1) \lambda_{1}$ 's times $1 / \mu_{M, P-1}$. Accordingly, the ratio of the second age group to the first at time $P$ (that is the $(2,1)$ element of $U_{M P}$ ) is given by

$$
\frac{\mu_{M P}}{\left(\lambda_{1 P} \mu_{M, P-1}\right)} .
$$

Equation (15) gives the size and structure of the long term "intrinsically dynamic model" (IDM) in terms of known PPM roots and the still to be determined mean age function $\mu_{M t}$. To find $\mu_{M t}$, note that the form of the column vector in equation (15) and the known constant subordinate eigenvector allow us to write

$$
U_{M t}=\left(\begin{array}{cc}
1 & 1 \\
\frac{\mu_{M t}}{\lambda_{1 t} \mu_{M, t-1}} & \frac{1}{\lambda_{2}}
\end{array}\right) .
$$

Because, at all times, $V=U^{-1}$, equation (16) can be used to find $V_{M t}$, and show that the scalar factor of $V_{M t}$ is

$$
\frac{1}{\mu_{M t}}=\frac{\lambda_{1 t}}{\lambda_{1 t}-\lambda_{2}\left(\frac{\mu_{M t}}{\mu_{M, t-1}}\right)} .
$$

Rewriting equation (17), we have the recursive relationship

$$
\frac{1}{\mu_{M t}}=\frac{\lambda_{1 t}+\frac{\lambda_{2}}{\mu_{M, t-1}}}{\lambda_{1 t}}
$$


Schoen: Intrinsically dynamic population models

at every time $t$. Combining equation (18) for times $t, t-1, t-2$, and so on to eliminate $\mu_{M}$ values at times before $t$ gives the solution

$$
\frac{1}{\mu_{M t}}=1+\frac{\lambda_{2}}{\lambda_{1 t}}+\frac{\lambda_{2}^{2}}{\lambda_{1 t} \lambda_{1, t-1}}+\frac{\lambda_{2}^{3}}{\lambda_{1 t} \lambda_{1, t-1} \lambda_{1, t-2}}+\frac{\lambda_{2}^{4}}{\lambda_{1 t} \lambda_{1, t-1} \lambda_{1, t-2} \lambda_{1, t-3}}+\ldots .
$$

The infinite series in equation (19) must converge because its terms alternate in sign (as $\lambda_{2}<0$ ) and the absolute value of the ratio $\lambda_{2} / \lambda_{1 t}$ is always less than 1 (since $\lambda_{1 t}$ is the dominant root). Straightforward algebra confirms that equations (15) and (19) satisfy the long term IDM projection equation

$$
x_{t}=A_{t} x_{t-1}
$$

when $x_{t}$ and $x_{t-1}$ are equated to the product of the $\lambda_{1}$ 's times the column vector given in equation (15), and $A_{t}$ is given by equation (10). In the stable case, where $\lambda_{1}$ is constant, the series in equation (19) sums to $\lambda_{1} /\left(\lambda_{1}-\lambda_{2}\right)$, the scalar factor $\frac{1}{\mu_{A}}$ in equation (9). [see Appendix note 1]

Heuristically, as time passes, equation (19) shows how a one time increase in $\lambda_{1}$ is steadily diminished by being relegated to successively smaller, higher order terms until its effect disappears. It follows that $\frac{1}{\mu_{M t}}$ increases, or the product matrix mean age at childbearing decreases, whenever $\lambda_{1 t}$ rises. Fundamentally, equation (19) reconciles population growth by $\lambda_{1 t}$ between times $t-1$ and $t$ with the population's legacy of past growth at different rates, growth which leaves its imprint in the population's age composition. The birth cohort size adjustments that follow from equation (19) make the long term "intrinsic dynamics" of the model possible.

The solution for the 2 age group IDM in equations (15) and (19) was verified by comparison with calculations on hypothetical data using the mathematical package Maple. Table 1 compares the product matrix with values calculated from equations (15) and (19). In the calculations, $\lambda_{1 t}=1+.04 t$ and $\lambda_{2}=-0.4$. Those values imply $a_{t}=.6+.04 t$ and $b_{t}=.4+.016 t$. The Net Reproduction Rate (NRR) at time $t, R_{t}$, is the sum of $a_{t}$ and $b_{t}$ or $1+.2 t$. After 12 projection intervals (300 years), the product matrix is close to rank one as $\lambda_{2}^{12}=.0000168$. By projection, mean age $\mu_{M, 12}=1.26871$ (in units of 25 years). Table 1 shows five different theoretically calculated values, where the series in equation (19) is followed to consecutively higher powers of $\lambda_{2}$. Convergence occurs fairly quickly, with 6 terms of the series (150 years) providing accuracy to about .002 and 9 terms to about .0001 .

We began the model development with the two age group case as that involves the simplest mathematics. However, the same constant subordinate eigenstructure/intrinsically dynamic approach generalizes to any number of age groups, as shown in the following sections. 
Table 1: Comparison of Two Age Group Intrinsically Dynamic Model Values From Equations (15) and (19) With Those From a Population Projection

\begin{tabular}{|c|c|c|c|c|c|c|}
\hline & \multirow{2}{*}{$\begin{array}{l}\text { Product matrix } \\
\text { element from } \\
\text { population } \\
\text { projection }\end{array}$} & \multicolumn{5}{|c|}{$\begin{array}{l}\text { Product matrix element from summing } \\
\text { the series up to } \lambda_{2} \text { terms of the power }\end{array}$} \\
\hline & & 5 & 6 & 7 & 8 & 9 \\
\hline Age group (j) & & & & & & \\
\hline 1 & 11.73411 & 11.72737 & 11.73626 & 11.73339 & 11.73435 & 11.73402 \\
\hline 2 & 7.88256 & 7.87718 & 7.88435 & 7.88196 & 7.88279 & 7.88249 \\
\hline
\end{tabular}

Note: Population projection matrix $\left(A_{t}\right)$ specified by $\lambda_{1 t}=1+.04 t$ and $\lambda_{2}=-0.4$.Values shown refer to the $(j, 1)$ element of the time 12 product matrix.

\section{The three age group IDM}

Let our 3 age group, time varying Leslie matrix, $A_{t}$, be given by

$$
A_{t}=\left(\begin{array}{ccc}
a_{t} & b_{t} & c_{t} \\
1 & 0 & 0 \\
0 & 1 & 0
\end{array}\right)
$$

where again we assume that mortality is included in the first row elements and our projection follows the birth trajectory. The eigenvectors of PPM $A_{t}$ are the roots of the characteristic equation (cf. Pollard 1973)

$$
\lambda^{3}-a_{t} \lambda^{2}-b_{t} \lambda-c_{t}=0
$$

and can readily be determined analytically using a mathematical program like Maple or Mathematica. [see Appendix note 2] Tuljapurkar (1993: 265) showed that the net maternity values of any Leslie matrix can be written in terms of its eigenvalues. Using that relationship, $A_{t}$ can be written

$$
A_{t}=\left(\begin{array}{ccc}
\lambda_{1 t}+\lambda_{2}+\lambda_{3} & -\left(\lambda_{1 t} \lambda_{2}+\lambda_{1 t} \lambda_{3}+\lambda_{2} \lambda_{3}\right) & \lambda_{1 t} \lambda_{2} \lambda_{3} \\
1 & 0 & 0 \\
0 & 1 & 0
\end{array}\right)
$$

where we again assume that only $\lambda_{1}$ varies with time.

From the constant subordinate eigenstructure restriction, we obtain the same constraints on the product of successive PPMs and on the eigenstructure of the product matrix as in the 2 age group case. At any time $\tau$, the right eigenvector matrix of 3 age group PPM 
Schoen: Intrinsically dynamic population models

$A_{\tau}$ is

$$
U_{\tau}=\left(\begin{array}{ccc}
1 & 1 & 1 \\
\lambda_{1 \tau}^{-1} & \lambda_{2}^{-1} & \lambda_{3}^{-1} \\
\lambda_{1 \tau}^{-2} & \lambda_{2}^{-2} & \lambda_{3}^{-2}
\end{array}\right)
$$

The inverse of $U \tau$ yields the left eigenvector matrix

$$
V_{\tau}=\left(\begin{array}{ccc}
1 & -\left(\lambda_{2}+\lambda_{3}\right) & \lambda_{2} \lambda_{3} \\
\frac{-\lambda_{2}^{2}\left(\lambda_{1 \tau}-\lambda_{3}\right)}{\lambda_{1 \tau}^{2}\left(\lambda_{2}-\lambda_{3}\right)} & \frac{\lambda_{2}^{2}\left(\lambda_{1 \tau}^{2}-\lambda_{3}^{2}\right)}{\lambda_{1 \tau}^{2}\left(\lambda_{2}-\lambda_{3}\right)} & \frac{-\lambda_{2}^{2} \lambda_{1 \tau} \lambda_{3}\left(\lambda_{1 \tau}-\lambda_{3}\right)}{\lambda_{1 \tau}^{2}\left(\lambda_{2}-\lambda_{3}\right)} \\
\frac{\lambda_{3}^{2}\left(\lambda_{1 \tau}-\lambda_{2}\right)}{\lambda_{1 \tau}^{2}\left(\lambda_{2}-\lambda_{3}\right)} & \frac{-\lambda_{3}^{2}\left(\lambda_{1 \tau}^{2}-\lambda_{2}^{2}\right)}{\lambda_{1 \tau}^{2}\left(\lambda_{2}-\lambda_{3}\right)} & \frac{\lambda_{3}^{2} \lambda_{1 \tau} \lambda_{2}\left(\lambda_{1 \tau}-\lambda_{2}\right)}{\lambda_{1 \tau}^{2}\left(\lambda_{2}-\lambda_{3}\right)}
\end{array}\right)\left\{\frac{1}{\mu_{A \tau}}\right\}
$$

where the mean age of childbearing implied by $A_{\tau}, \mu_{A \tau}$, is given by

$$
\mu_{A \tau}=\frac{\left(\lambda_{1 \tau}-\lambda_{2}\right)\left(\lambda_{1 \tau}-\lambda_{3}\right)}{\lambda_{1 \tau}^{2}} .
$$

Using the structure of the eigenvectors of $A_{\tau}$ and the reasoning underlying equation (15), we can write the long term product matrix as

$$
M_{0, t}=\left\{\prod_{j=1}^{t} \lambda_{1 j}\right\}\left(\begin{array}{c}
\frac{1}{\mu_{M t}} \\
\frac{1}{\left(\lambda_{1 t} \mu_{M, t-1}\right)} \\
\frac{1}{\left(\lambda_{1 t} \lambda_{1, t-1} \mu_{M, t-2}\right)}
\end{array}\right)\left(\begin{array}{lll}
1 & -\left(\lambda_{2}+\lambda_{3}\right) & \left.\lambda_{2} \lambda_{3}\right)
\end{array}\right.
$$

The $(1,1)$ element of $M_{0, t}$ is the product of the individual PPM growth rates, divided by $\mu_{M t}$; the $(2,1)$ element is the product of the PPMs up to time $t-1$, divided by $\mu_{M, t-1}$; and the $(3,1)$ element is the product of the PPMs up to time $t-2$, divided by $\mu_{M, t-2}$. The latter two elements are simply the $(1,1)$ elements of $M$ at times $t-1$ and $t-2$, respectively. The row vector of relative reproductive values remains fixed over time and equal to its stable population counterpart.

To find $\mu_{M t}$, we note that with constant subordinate eigenvectors the full $U_{M t}$ matrix can be written

$$
U_{M t}=\left(\begin{array}{ccc}
1 & 1 & 1 \\
\frac{\mu_{M t}}{\left(\lambda_{1 t} \mu_{M, t-1}\right)} & \lambda_{2}^{-1} & \lambda_{3}^{-1} \\
\frac{\mu_{M t}}{\left(\lambda_{1 t} \lambda_{1, t-1} \mu_{M, t-2}\right)} & \lambda_{2}^{-2} & \lambda_{3}^{-2}
\end{array}\right)
$$


Using the relationship $V_{M t}=U_{M t}^{-1}$ and equating the scalar factor associated with the first row of $V_{M t}$ to $1 / \mu_{M t}$ yields the recursive relationship

$$
\frac{1}{\mu_{M t}}=1+\frac{\left(\lambda_{2}+\lambda_{3}\right)}{\lambda_{1 t} \mu_{M, t-1}}-\frac{\left(\lambda_{2} \lambda_{3}\right)}{\lambda_{1 t} \lambda_{1, t-1} \mu_{M, t-2}} .
$$

Using the relationship in equation (29) to eliminate $\mu_{M}$ at times before t leads to $\frac{1}{\mu_{M t}}$ as the sum of the series

$$
\begin{aligned}
\frac{1}{\mu_{M t}}=1 & +\frac{\left(\lambda_{2}+\lambda_{3}\right)}{\lambda_{1 t}}+\frac{\frac{\left(\lambda_{2}^{3}-\lambda_{3}^{3}\right)}{\left(\lambda_{2}-\lambda_{3}\right)}}{\left(\lambda_{1 t} \lambda_{1, t-1}\right)}+ \\
& +\frac{\frac{\left(\lambda_{2}^{4}-\lambda_{3}^{4}\right)}{\left(\lambda_{2}-\lambda_{3}\right)}}{\left(\lambda_{1 t} \lambda_{1, t-1} \lambda_{1, t-2}\right)}+\frac{\frac{\left(\lambda_{2}^{5}-\lambda_{3}^{5}\right)}{\left(\lambda_{2}-\lambda_{3}\right)}}{\left(\lambda_{1 t} \lambda_{1, t-1} \lambda_{1, t-2} \lambda_{1, t-3}\right)}+\ldots
\end{aligned}
$$

Equations (27) and (30) provide the basic relationships underlying the 3 age group IDM. The infinite series in equation (30) converges because it is an alternating series and $\lambda_{1 t}>-\left(\lambda_{2}+\lambda_{3}\right)$, since $\lambda_{1 t}+\lambda_{2}+\lambda_{3}=a_{t}>0$. Equations (23), (27), and (30) satisfy basic projection equation (20), confirming the solution. Those equations apply regardless of whether the two subordinate roots are real and unequal, real and equal, or complex conjugates.

Table 2 compares the projected product matrix with values calculated from equations (27) and (30). In the calculations, $\lambda_{1 t}=1+.02 t, \lambda_{2}=-.2$, and $\lambda_{3}=-.5$. The NRR at any time can be found by summing the first row elements of the $A_{t}$ matrix in equation (21). Algebraically, that yields

$$
R_{t}=1-\left(1-\lambda_{1 t}\right)\left(1-\lambda_{2}\right)\left(1-\lambda_{3}\right) .
$$

With the present values, $R_{t}=1+.036 t$, indicating how the NRR increases linearly with time (and $\lambda_{1 t}$ ). After 20 projection intervals (300 years, as 3 age groups imply 15 year intervals), the product matrix is very close to rank one; $\lambda_{2}^{20}$ is less than .000001. In the projection, mean age $\mu_{M, 20}=1.54841$ (in units of 15 years). Table 2 shows four theoretically calculated values, where the series in equation (30) is summed over the first 7 through 10 terms. Convergence is a bit slower (in terms of number of intervals) than in the two age group case. Differences between projected and calculated values are about $.01-.02$ after 7 terms of the series, and about .001 after 10 terms (150 years).

Table 3 looks at four different fertility patterns and how their shape varies with the size of the dominant root. The two subordinate roots specify the age pattern of fertility. Here, Pattern 1 emphasizes early fertility, Pattern 2 mid-reproductive age fertility, and Patterns 3 and 4 later age fertility. Pattern 2 involves 2 real roots, Patterns 1 and 4 complex conjugate 
Schoen: Intrinsically dynamic population models

Table 2: Comparison of Three Age Group Intrinsically Dynamic Model Values From Equations (27) and (30) With Those From a Population Projection

\begin{tabular}{|c|c|c|c|c|c|}
\hline & \multirow{2}{*}{$\begin{array}{l}\text { Product matrix } \\
\text { element from } \\
\text { population projection }\end{array}$} & \multicolumn{4}{|c|}{$\begin{array}{l}\text { Product matrix element from equations } \\
\text { (27) and (30), summing the series to term number }\end{array}$} \\
\hline & & 7 & 8 & 9 & 10 \\
\hline \multicolumn{6}{|l|}{ Age group (j) } \\
\hline 1 & 26.67127 & 26.65159 & 26.67917 & 26.66805 & 26.67261 \\
\hline 2 & 18.94539 & 18.92959 & 18.95184 & 18.94272 & 18.94652 \\
\hline 3 & 13.65055 & 13.63766 & 13.65589 & 13.64830 & 13.65152 \\
\hline
\end{tabular}

Note: Population projection matrix $\left(A_{t}\right)$ specified by $\lambda_{1 t}=1+.02 t, \lambda_{2}=-.2$ and $\lambda_{3}=-.5$. Values shown refer to the $(j, 1)$ element of the time 20 product matrix.

roots, and Pattern 3 a double root. Those four patterns span the most commonly found three age group discrete fertility schedules (Schoen and Kim 1996).

Table 3: Fertility Patterns and Their Variation With the Dominant Root in 3 Age Group Intrinsically Dynamic Models

\begin{tabular}{ccccccccccccc}
\hline & \multicolumn{3}{c}{ Pattern 1 } & \multicolumn{3}{c}{ Pattern 2 } & \multicolumn{3}{c}{ Pattern 3 } & \multicolumn{3}{c}{ Pattern 4 } \\
Root $\left(\lambda_{1}\right)$ & $\mathrm{a}$ & $\mathrm{b}$ & $\mathrm{c}$ & $\mathrm{a}$ & $\mathrm{b}$ & $\mathrm{c}$ & $\mathrm{a}$ & $\mathrm{b}$ & $\mathrm{c}$ & $\mathrm{a}$ & $\mathrm{b}$ & $\mathrm{c}$ \\
\hline .8 & .2 & .38 & .08 & .1 & .46 & .08 & 0 & .48 & .128 & 0 & .44 & .16 \\
.9 & .3 & .44 & .09 & .2 & .53 & .09 & .1 & .56 & .144 & .1 & .52 & .18 \\
1.0 & .4 & .5 & .1 & .3 & .6 & .1 & .2 & .64 & .16 & .2 & .6 & .2 \\
1.1 & .5 & .56 & .11 & .4 & .67 & .11 & .3 & .72 & .176 & .3 & .68 & .22 \\
1.2 & .6 & .62 & .12 & .5 & .74 & .12 & .4 & .80 & .192 & .4 & .76 & .24 \\
1.3 & .7 & .68 & .13 & .6 & .81 & .13 & .5 & .88 & .208 & .5 & .84 & .26 \\
1.4 & .8 & .74 & .14 & .7 & .88 & .14 & .6 & .96 & .224 & .6 & .92 & .28 \\
1.5 & .9 & .80 & .15 & .8 & .95 & .15 & .7 & 1.04 & .24 & .7 & 1.00 & .30 \\
Subordinate roots & $-.3 \pm .1 i$ & \multicolumn{4}{c}{$-.2,-.5$} & -.4 (double root) & & $-.4 \pm .2 i$ \\
\hline
\end{tabular}

Table 3 shows how in every pattern, at every age, fertility changes linearly with $\lambda_{1}$. The amount of change in an age-specific rate varies across patterns and ages, and follows the expressions shown in the first row of the PPM in equation (23). Increases in $\lambda_{1}$ lead to ages 0-14 having the largest increases, ages 15-29 the second largest, and ages 30-44 the smallest. Thus as $\lambda_{1}$ increases, the mean age of childbearing decreases. That pattern of change departs from proportionality, but for the most part the fertility schedules are plausible given the observed variability in human fertility. Recent low fertility in the West, for example, has been accompanied by rising mean ages at childbearing. However, Pattern 1 has unusually high relative fertility levels at ages 0-14 for $\lambda_{1}=1.5$ (i.e. when Lotka's $r=.027$ ), and Patterns 3 and 4 have zero fertility at ages $0-14$ when $\lambda_{1}=.8$ (i.e. $r=-.015$ ). Because the rate in the first age group mirrors $\lambda_{1}$ in its movements up 
and down, values of $\lambda_{1}$ below .8 would lead to unacceptable (negative) fertility values.

The constant subordinate eigenstructure assumption was chosen for its mathematical, not its demographic, properties. By restricting the age pattern of fertility, it permits any pattern of change in fertility levels over time and allows the resultant birth trajectory to be found (and, with a mortality assumption, gives the age composition of the population at any time). The ability to vary the level of fertility represents a substantial extension of the stable population model, and at the price of an assumption that is no more unrealistic than fixed rates. The IDM first row PPM elements are all linear functions of $\lambda_{1}$, and hence of each other, and need not reproduce (or approximate) the characteristic age pattern of fertility. Yet they generally produce reasonable values over a broad range of $\lambda$ values when there are three reproductive ages. While investigators using IDMs need to verify that acceptable age patterns of fertility exist, Table 3 suggests that it is usually possible to do so.

\section{The $n$ age group IDM}

To extend the intrinsically dynamic model to the case of $n$ age groups, let our standard form Leslie matrix $A_{t}$ have first row elements $a_{j t}, j=1, \ldots, n$. As before, the subdiagonal elements are equal to 1 . The characteristic equation of $A_{t}$ is then

$$
\lambda_{t}^{n}-a_{1 t} \lambda_{t}^{n-1}-a_{2 t} \lambda_{t}^{n-2}-\ldots-a_{n t}=0 .
$$

From the relationship between roots and coefficients in polynomial equations, the $n$ coefficients can be written in terms of the $n$ roots of equation (32). Specifically, $a_{1 t}$ is the sum of the roots taken one at a time; $a_{2 t}$ is minus the sum of the roots taken 2 at a time; and $a_{j t}$ is $(-1)^{j+1}$ times the sum of the roots taken $j$ at a time. As a result, in the IDM all of the $a_{j t}$ are linear functions of $\lambda_{1 t}$.

In Leslie matrix $A_{t}$, several basic demographic measures are expressible as relatively simple functions of all $n$ roots. Summing the first row elements of the matrix and rearranging terms gives the NRR as

$$
R_{t}=1-\prod_{j=1}^{n}\left(1-\lambda_{j t}\right) .
$$

In intrinsically dynamic models, the NRR is always a linear function of $\lambda_{1}$ (and vice versa).

The mean age of childbearing implied by $A_{t}$ can be defined by the relationship

$$
\mu_{A t}=\frac{a_{1 t}}{\lambda_{1 t}}+\frac{2 a_{2 t}}{\lambda_{1 t}^{2}}+\ldots+\frac{n a_{n t}}{\lambda_{1 t}^{n}} .
$$


Schoen: Intrinsically dynamic population models

Rewriting that relationship in terms of roots yields

$$
\mu_{A t}=\left(\lambda_{1 t}^{1-n}\right) \prod_{j=2}^{n}\left(\lambda_{1 t}-\lambda_{j}\right) .
$$

The approach used in the 2 and 3 age group models can generate the product matrix and $\mu_{M t}$ in the $n$ age group case. Once again, the $j$ th root of the product matrix is the product of the $j$ th roots of the individual PPMs. Apart from the $\mu_{M t}$ factor and with one as the first element, the $j$ th element in the first row of the left eigenvector matrix is $(-1)^{j-1}$ times the sum of the subordinate roots taken $j-1$ at a time. The first column of the right eigenvector matrix has first term $1 / \mu_{M t}$, and $j$ th term

$$
\frac{1}{\left[\mu_{M, t-j+1}\left(\prod \lambda_{1 j}\right)\right]},
$$

where the product goes from $t-j+2$ to $t$. A recursive equation for mean age $\mu_{M t}$ can be found by extending equation (28) to specify the first column of $U_{M t}$, using $V_{M t}=U_{M t}^{-1}$, and then equating the scalar factor of $V_{M t}$ to $1 / \mu_{M t}$. As $n$ becomes large, the expressions get quite complicated.

Beyond complexity, there is a serious problem with the $n$ age group IDM as a vehicle for analyzing human populations. The pattern of fertility change required by the constant subordinate eigenstructure assumption becomes increasingly inappropriate when $n \geq 4$. Consider the conventional Leslie matrix with 10 five-year age groups. Since human populations have essentially zero fertility below age 10 , the $(1,1)$ element of that Leslie matrix must be zero. However, any change in the dominant root would cause the $(1,1)$ element to change by an equal amount, producing a PPM with unrealistic or unacceptable values. Consequently, the focus here is on the 3 age group model. Table 3 shows that the age patterns of fertility are quite reasonable in that model, and 15 year age groups coincide closely with the typical beginning of reproduction, the mean age of childbearing, and the end of reproduction. Moreover, it is not difficult to condense a 10 age group Leslie matrix to 3 groups (Keyfitz 1968, p37-40).

\section{IDM Dynamics}

\subsection{The IDM birth trajectory}

The number of births at time $t$ (or more precisely the number of persons in the first age group at that time) can be found by dividing $\lambda_{M t}$, the product of the PPM growth rates (or the cumulative growth rate up to time $t$ ), by $\mu_{M t}$. When the $\lambda_{1 t}$ have a known functional form, $\lambda_{M t}$ can often be found analytically. 
Consider the case where $\lambda_{1 t}=e^{h t}$, for some known constant $h$. Since

$$
\sum_{j=1}^{t} h j=h \frac{t(t+1)}{2}
$$

we have

$$
\lambda_{M t}=\exp \left(\frac{h t(t+1)}{2}\right) .
$$

Using different assumptions, Schoen and Kim (1997) and Schoen and Jonsson (2003) also found exponentiated quadratic growth associated with exponentially increasing net reproduction. Other polynomial functions for $\lambda_{1 t}$ also lead to exponentiated growth in births at an order one degree higher than the largest power of $\mathrm{t}$ in $\lambda_{1 t}$. For example, Maple calculations indicate that if $\lambda_{1 t}=\exp \left(h t^{2}\right)$, then $\lambda_{M t}=\exp \left(\frac{h t(t+1)(2 t+1)}{6}\right)$, and if $\lambda_{1 t}=\exp \left(h t^{3}\right)$, then $\lambda_{M t}=\exp \left(\frac{h t^{2}(t+1)^{2}}{4}\right)$.

The ability to analyze cyclical fluctuations in fertility is particularly useful, and sinusoidal patterns in $\lambda_{1 t}$ can be summed analytically. In particular, Maple computations show that

$$
\sum_{j=1}^{t} \sin \omega j=\frac{1}{2}\left[\sin \omega-\sin \omega(t+1)+\left(\cot \frac{\omega}{2}\right)\{\sin \omega \cos \omega-\cos \omega(t+1)\}\right] .
$$

Schoen and Kim (1997) found a similar expression for cyclical net reproduction by assuming a constant generation length, though their approach did not provide any underlying age-specific birth rates. Under polynomial growth in $\lambda_{1 t}$, the relative size adjustments produced by the $1 / \mu_{M t}$ factor may be small, as they were in the case of the linearly increasing $\lambda_{1 t}$ of Table 2 . With cyclical changes, however, those adjustments may play a much larger role.

\subsection{Observed populations as IDM populations}

Any observed population distribution can be viewed as an IDM population distribution. That representation is not unique, however, as different intrinsically dynamic models follow from alternative assumptions regarding the (constant) subordinate roots and the nature of the past birth trajectory.

Consider the case of an arbitrary initial population, $x_{0}$, were we focus on the 3 reproductive age groups and scale the population by assuming that there is one person at ages $0-14$. In the absence of mortality, or after adjustments for mortality have been made to 
Schoen: Intrinsically dynamic population models

restore the birth sequence, we can write

$$
x_{0}=\left(\begin{array}{c}
1 \\
x_{20} \\
x_{30}
\end{array}\right)
$$

where $x_{j 0}$ represents the number of persons in the $j$ th age group at initial time 0 . To simplify the calculations, assume that the population was stable with dominant growth rate $\lambda_{A}$ and subordinate roots $\lambda_{2}$ and $\lambda_{3}$ before time $(-1)$. Then, from the definition of $\lambda_{A}$ and equation (26)

$$
\begin{aligned}
\lambda_{A} & =\frac{x_{20}}{x_{30}} \\
\mu_{A} & =\frac{\left(\lambda_{A}-\lambda_{2}\right)\left(\lambda_{A}-\lambda_{3}\right)}{\lambda_{A}^{2}} .
\end{aligned}
$$

To specify the IDM, we need to find the time 0 cumulative growth rate $\left(\lambda_{M_{0}}\right)$ and the time 0 product matrix mean age at childbearing $\left(\mu_{M 0}\right)$. Using equation (27), with the scale readjusted so that the population age $0-14$ is 1 , we can equate the second element of the scaled column vector with the second element in the population in equation (38) and write

$$
x_{20}=\frac{\mu_{M 0}}{\left(\mu_{A} \lambda_{M 0}\right)} .
$$

Using equations (30) and (39), we can replace the infinite series in equation (30) with the stable $\mu_{A}$ and show that

$$
\frac{1}{\mu_{M 0}}=1-\frac{\lambda_{A}}{\lambda_{M 0}\left(1-\frac{1}{\mu_{A}}\right)} .
$$

Combining equations (40) and (41) yields the solutions

$$
\begin{aligned}
& \lambda_{M 0}=\frac{1+x_{20} \lambda_{A}\left(\mu_{A}-1\right)}{x_{20} \mu_{A}} \\
& \mu_{M 0}=1+x_{20} \lambda_{A}\left(\mu_{A}-1\right) .
\end{aligned}
$$

With equation (42), the IDM representation can be written as

$$
x_{0}=\left(\begin{array}{c}
1 \\
{\left[\lambda_{A}+\mu_{A}\left(\lambda_{M 0}-\lambda_{A}\right)\right]^{-1}} \\
{\left[\lambda_{A}^{2}+\lambda_{A} \mu_{A}\left(\lambda_{M 0}-\lambda_{A}\right)\right]^{-1}}
\end{array}\right) .
$$


The form of equation (43) makes it clear that the age composition becomes that of the $\lambda_{A}$ stable population when $\lambda_{A}=\lambda_{M 0}$.

To illustrate the procedure numerically, let $x_{0}$ ' $=\left[\begin{array}{ll}1 & 6 \\ 6 & 6.4\end{array}\right]$, where the prime (') indicates the vector transpose. Assuming stability before time $(-1)$ and setting $\lambda_{2}=-.2$ and $\lambda_{3}=-.5$, we find $\lambda_{A}=1.5, \mu_{A}=1.51111, \lambda_{M 0}=1.61029$, and $\mu_{M 0}=1.46$, with age and time in units of 15 years. The faster pace of growth from time $(-1)$ to 0 $\left(\lambda_{0}=1 / .6=1.66667\right)$, compared to $\lambda_{A}=1.5$ between times $(-2)$ and $(-1)$, leads to an increase in cumulative growth from 1.5 to 1.61029 and a fall in the (implicit) product matrix mean age at childbearing from 1.51111 to 1.46 .

\subsection{Transitions between stable population regimes}

Intrinsically dynamic models can be used to examine transitions from one set of fixed rates to another. If a stable population growing at $\lambda_{A}$ were suddenly to shift to a regime with long term growth at $\lambda_{B}$, the subordinate roots remaining constant, its dynamics would be that of an IDM.

Consider a 3 reproductive age group model, and let the number of persons under age 15 at time 0 be scaled to 1 . With the change in regimes from $\lambda_{A}$ to $\lambda_{B}$ occurring at time 0 , equations (27) and (30) describe the subsequent behavior of the population. Table 4 and Figure 1 show the birth trajectory (i.e. the number of persons under age 15) when $\lambda_{A}=1.5$ and $\lambda_{B}=1$ under two different patterns of fertility, specifically Patterns 2 and 4 of Table 3. After the fall in fertility to replacement level, the number of births falls sharply, then recovers some, and eventually approaches its ultimate stationary level. When all of the $\lambda_{1}$ values in equation (30) are the same, equation (26) applies. With the initial number of births scaled to 1, equation (27) indicates that the ultimate birth level is $\frac{\mu_{A}}{\mu_{B}}$. Table 4 and Figure 2 show how $\mu_{M t}$ moves from $\mu_{A}$ to $\mu_{B}$ along a path similar to that of the birth trajectory. Consistent with Preston (1986) and Kim and Schoen (1997), the number of persons under age 30 remains quite level during the transition.

The concept of population momentum, introduced in Keyfitz (1971), refers to the increase in population size that accompanies the transition to zero growth. Given a drop to replacement level fertility at a specified initial time, momentum, $\Omega$, is the ratio of the total size of the ultimate stationary population to the total size of the initial population. Mathematically, it can be written (cf. Schoen and Jonsson 2003)

$$
\Omega=b e_{0} Q
$$

where $b$ is the birth rate of the initial population, $e_{0}$ is the life expectancy at birth in the stationary population, and $Q$ is the size of the ultimate birth cohort relative to that of the 
Schoen: Intrinsically dynamic population models

Figure 1: The Birth Trajectory Following a Transition, at Time 0, From a Stable $\left(\lambda_{A}=1.5\right)$ to a Stationary $\left(\lambda_{B}=1\right)$ Regime, Under Two Fertility Patterns

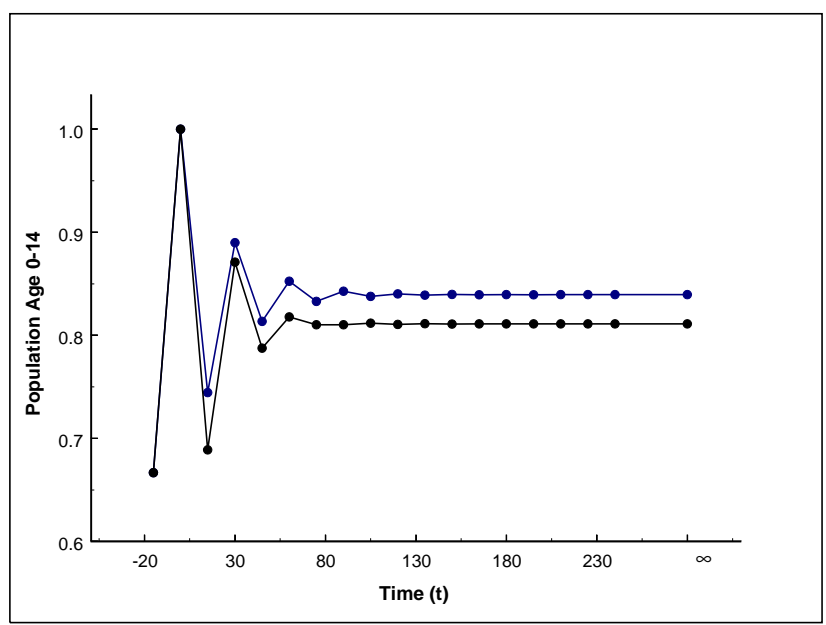

Figure 2: The Cumulative Mean Age of Childbearing $\left(\mu_{M t}\right)$ Following a Transition, at Time 0, From a Stable $\left(\lambda_{A}=1.5\right)$ to a Stationary $\left(\lambda_{B}=1\right)$ Regime, Under Two Fertility Patterns

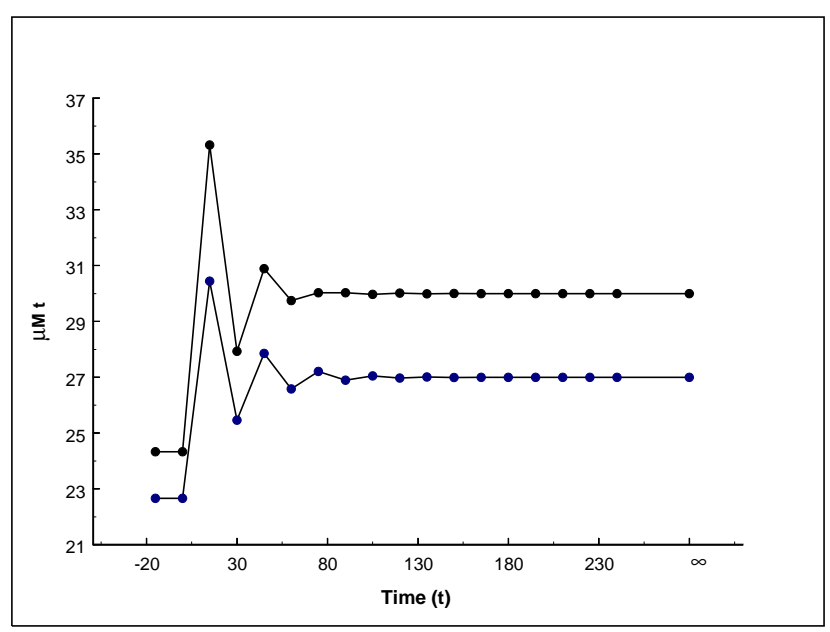


Table 4: The Birth Trajectory and Related Measures Following a Transition, at Time 0, From a Stable $\left(\lambda_{A}=1.5\right)$ to a Stationary $\left(\lambda_{B}=1\right)$ Regime, Under Two Age Patterns of Fertility

\begin{tabular}{|c|c|c|c|c|c|c|}
\hline \multirow{2}{*}{$\begin{array}{l}\text { Time }(\mathrm{t}) \\
\text { [in years] }\end{array}$} & \multicolumn{2}{|c|}{$\begin{array}{l}\text { Number of persons } \\
\text { aged } 0-14 \text { years }\end{array}$} & \multicolumn{2}{|c|}{$\begin{array}{l}\text { Number of persons } \\
\text { aged } 0-29 \text { years }\end{array}$} & \multicolumn{2}{|c|}{$\begin{array}{c}\text { Product matrix mean } \\
\text { age of childbearing }\left(\mu_{M t}\right)\end{array}$} \\
\hline & Pattern 2 & Pattern 4 & Pattern 2 & Pattern 4 & Pattern 2 & Pattern 4 \\
\hline 0 & 1 & 1 & 1.66667 & 1.66667 & $22.66667\left(\mu_{A}\right)$ & $24.33333\left(\mu_{A}\right)$ \\
\hline 15 & .74444 & 68889 & 1.74444 & 1.68889 & 30.44776 & 35.32258 \\
\hline 30 & .89000 & .87111 & 1.63444 & 1.56000 & 25.46816 & 27.93367 \\
\hline 45 & .81367 & .78756 & 1.70367 & 1.65867 & 27.85743 & 30.89729 \\
\hline 60 & .85254 & 81796 & 1.66621 & 1.60551 & 26.58708 & 29.74897 \\
\hline 75 & .83296 & .81035 & 1.68551 & 1.62830 & 27.21208 & 30.02830 \\
\hline 90 & .84278 & .81035 & 1.67575 & 1.62070 & 26.89504 & 30.02804 \\
\hline 105 & .83787 & .81187 & 1.68065 & 1.62222 & 27.05281 & 29.97196 \\
\hline 120 & .84033 & .81066 & 1.67819 & 1.62252 & 26.97365 & 30.01686 \\
\hline 135 & .83910 & .81132 & 1.67942 & 1.62198 & 27.01317 & 29.99213 \\
\hline 150 & .83971 & .81103 & 1.67881 & 1.62236 & 26.99338 & 30.00292 \\
\hline 165 & .83940 & .81113 & 1.67912 & 1.62216 & 27.00326 & 29.99922 \\
\hline 180 & .83956 & .81111 & 1.67896 & 1.62224 & 26.99829 & 30.00001 \\
\hline 195 & .83948 & .81111 & 1.67904 & 1.62222 & 27.00073 & 30.00011 \\
\hline 210 & .83952 & .81111 & 1.67901 & 1.62222 & 26.99945 & 29.99986 \\
\hline 225 & .83951 & .81111 & 1.67903 & 1.62222 & 27.00000 & 30.00001 \\
\hline 240 & .83952 & .81111 & 1.67903 & 1.62222 & 26.99959 & 29.99990 \\
\hline$\infty$ & .83951 & .81111 & 1.67901 & 1.62222 & $27.00000\left(\mu_{B}\right)$ & $30.00000\left(\mu_{B}\right)$ \\
\hline
\end{tabular}

Note: Equation (30) is summed through the $16^{\text {th }}$ term. Under Fertility Pattern $2, \lambda_{2}=-.2$ and $\lambda_{3}=-.5$. Under Fertility Pattern $4, \lambda_{2}=-.4+.2 i$ and $\lambda_{3}=-.4-.2 i$.

initial birth cohort. As indicated above, in the IDM

$$
Q=\frac{\mu_{A}}{\mu_{B}} .
$$

When fertility falls, the mean age at childbearing in an IDM rises. The ratio of initial to ultimate mean ages of childbearing mirrors the ratio of initial to ultimate birth cohort sizes.

\subsection{Momentum following a gradual or irregular decline to zero growth}

Birth cohort size adjustment $Q$ can, more generally, be thought of as the change in relative cohort size associated with changes in vital rates, after removing the effect of PPM growth rates. In the context of intrinsically dynamic models, that $Q$ can be expressed quite simply. Equation (27) shows that the birth cohort size change from time $A$ to time $B$ is always given by $\mu_{M A} / \mu_{M B}$, the ratio of the product matrix mean ages at childbearing. 
That relationship, made applicable to any initial population by equation (42), means that the IDM context can be used to examine the population growth associated with any arbitrary route to stationarity. The subject has received considerable attention recently for several reasons. Substantively, Bongaarts and Bulatao (1999) found that momentum effects are likely to account for most of the future growth in the world's population. Analytically, Schoen and Kim (1998), Li and Tuljapurkar (1999; 2000), Goldstein (2002), Goldstein and Stecklov (2002), and Schoen and Jonsson (2003) have extended techniques for finding momentum under a variety of paths to zero growth. That work has demonstrated the dramatic increases in ultimate population size that result from delays in achieving zero growth. All of those approaches, however, are either approximate, impose substantial constraints on the length or pattern of decline, or both.

Equation (27) can yield exact solutions for essentially any path to zero growth in terms of initial mean age $\mu_{M 0}$, ultimate stationary mean age $\mu_{B}$, and the product of the nonstationary PPM growth rates between initial time 0 and the time, $B$, when the rates attain their final stationary level. Mathematically, with $Q^{*}$ denoting the relative size of the ultimate birth cohort,

$$
Q^{*}=\left\{\prod_{j=1}^{B} \lambda_{1 j}\right\} \frac{\mu_{M 0}}{\mu_{B}} .
$$

In the intrinsically dynamic population, ultimate birth cohort size is determined by the product of individual PPM intrinsic growth rates, modified only by the initial and ultimate product matrix mean ages at childbearing. Momentum follows immediately from equation (44).

Table 5 compares population momentum values from equations (44) and (46) with those from population projections under a range of assumptions about the length and pattern of fertility decline. The initial population is the IDM with $\lambda_{1}=1.5, \lambda_{2}=-.2$, and $\lambda_{3}=-.5$, which has an NRR of 1.90 . Under contemporary conditions, the value of $b e_{0}$ for that population is approximately 2 , and that value is used in the calculations. Two different patterns of decline over time are considered, one linear with respect to the NRR, and the other linear with respect to $\lambda_{1}$. One projection is done assuming a linear (proportional) decline over age, and the other assuming that the pattern of decline keeps the subordinate eigenvalues constant.

With a linear decline in NRR over time, equation (46) yields values that are identical to those from the population projection that assumed an IDM pattern of decline over age. The projection assuming a linear (proportional) decline over age produced similar figures, though the gap widened somewhat as the period of delay increased from 0 to 45 years. In an IDM, $\lambda_{1}$ is a linear transformation of the NRR. Thus, in the case of linear declines in growth, the IDM equations and projection yield values identical to those found in the 
Table 5: Population Momentum Associated With Different Routes to Stationarity, Calculated By Population Projections and By Equations (44) and (46)

\begin{tabular}{|c|c|c|c|c|c|c|}
\hline \multirow{3}{*}{$\begin{array}{l}\text { Years Before } \\
\text { Zero Growth } \\
\text { Attained }\end{array}$} & \multicolumn{3}{|c|}{ Linear Decline in NRR Over Time } & \multicolumn{3}{|c|}{ Linear Decline in PPM $\lambda_{1}$ Over Time } \\
\hline & \multicolumn{2}{|c|}{ From Projection } & \multirow[b]{2}{*}{$\begin{array}{l}\text { From IDM } \\
\text { equation (46) }\end{array}$} & \multicolumn{2}{|c|}{ From Projection } & \multirow[b]{2}{*}{$\begin{array}{l}\text { From IDM } \\
\text { equation (46) }\end{array}$} \\
\hline & $\begin{array}{l}\text { Linear decline } \\
\text { over age }\end{array}$ & $\begin{array}{l}\text { IDM decline } \\
\text { over age }\end{array}$ & & $\begin{array}{l}\text { Linear decline } \\
\text { over age }\end{array}$ & $\begin{array}{l}\text { IDM decline } \\
\text { over age }\end{array}$ & \\
\hline 0 & 1.714 & 1.679 & 1.679 & 1.714 & 1.679 & 1.679 \\
\hline 15 & 2.143 & 2.099 & 2.099 & 2.128 & 2.099 & 2.099 \\
\hline 30 & 2.678 & 2.612 & 2.612 & 2.645 & 2.612 & 2.612 \\
\hline 45 & 3.344 & 3.247 & 3.247 & 3.285 & 3.247 & 3.247 \\
\hline
\end{tabular}

Note: Initial population stable at $\lambda_{1}=1.5$ with $\mathrm{NRR}=1.90$. IDM subordinate roots are $\lambda_{2}=-.2$ and $\lambda_{3}=-.5$. In all models, the product of the initial birth rate and the ultimate life expectancy is set at 2 .

case of linear NRR declines. Because that NRR/growth relationship does not hold when fertility changes proportionally over all ages, the linear decline in $\lambda_{1}$ projections yield slightly different results from the linear decline in NRR projections. The rather modest effect associated with different age patterns of fertility decline can be seen as enhancing the value of equations (44) and (46), as it suggests that the age pattern required by the constant subordinate root assumption has only a small effect on momentum. In addition to validating the analytical results presented, Table 5 shows how delays in attaining zero growth greatly increase momentum. In the case considered, the population would grow from 1 to 1.7 if stationary rates were achieved immediately, but would grow to over 3.2 if the decline took place over 45 years.

\section{Summary and conclusions}

Intrinsically dynamic models, based on an assumption of constant subordinate roots, have been developed to provide a new approach to analyzing populations with changing rates. Because of the constraints on the age pattern of fertility associated with that assumption, attention is focused on the discrete (Leslie) model with 15 year age intervals. Equations (27) and (30) provide a complete solution for the population produced by any time pattern of change in the level of fertility (or in period-by-period growth levels).

The IDM provides a new generalization of the stable population model. Any observed population can be represented as an IDM, and the structure of IDMs greatly simplifies modeling the transition between any two stable regimes and assessing the momentum implications of delays in achieving replacement level fertility. 
Schoen: Intrinsically dynamic population models

\section{References}

Bennett, N.G. and S. Horiuchi. (1981). Estimating the completeness of death registration in a closed population. Population Index, 47, 207-21.

Birkhoff, G. and S. MacLane. (1959). A survey of modern algebra (rev. ed). New York: Macmillan.

Bongaarts, J. and R.A. Bulatao. (1999). Completing the demographic transition. Population and Development Review, 25, 515-29.

Caswell, H. (2001). Matrix population models (2d ed). Sunderland MA: Sinauer.

Coale, A.J. (1972). The growth and structure of human populations. Princeton: Princeton University Press.

Gantmacher, F.R. (1959). Matrix theory. New York: Chelsea.

Goldstein, J.R. (2002). Population momentum for gradual demographic transitions: An alternative approach. Demography, 39, 65-73.

Goldstein, J.R. and G. Stecklov. (2002). Long-range population projections made simple. Population and Development Review, 28, 121-41.

Keyfitz, N. (1968). Introduction to the mathematics of population. Reading MA: Addison-Wesley.

Keyfitz, N. (1971). On the momentum of population growth. Demography, 8, 71-80.

Kim, Y.J. (1987). Dynamics of populations with changing rates: Generalization of stable population theory. Theoretical Population Biology, 31, 306-22.

Kim, Y.J. and R. Schoen. (1993). On the intrinsic force of convergence to stability. Mathematical Population Studies, 4, 89-102.

Kim, Y.J. and R. Schoen. (1996). Populations with quadratic exponential growth. Mathematical Population Studies, 6, 19-33.

Kim, Y.J. and R. Schoen. (1997). Population momentum expresses population aging. Demography, 34, 421-27.

Land, K.C. and A. Rogers. (1982). Multidimensional mathematical demography. New York: Academic Press. 
Lee, R. (1974). The formal dynamics of controlled populations and the echo, the boom, and the bust. Demography, 11, 563-85.

Leslie, P.H. (1945). On the use of matrices in certain population mathematics. Biometrika, 33, 183-212.

Li, N. and S. Tuljapurkar. (1999). Population momentum for gradual demographic transitions. Population Studies, 53, 255-62.

Li, N. and S. Tuljapurkar. (2000). The solution of time-dependent population models. Mathematical Population Studies, 7, 311-29.

Lotka, A.J. (1939). Theorie analytique des associations biologiques. Paris: Hermann.

Pollard, J.H. (1973). Mathematical models for the growth of human populations. Cambridge: Cambridge University Press.

Preston, S.H. (1986). The relation between actual and intrinsic growth rates. Population Studies, 40, 495-501.

Preston, S.H. and A.J. Coale. (1982). Age structure, growth, attrition, and accession: A new synthesis. Population Index, 48, 217-59.

Rogers, A. (1975). Introduction to multiregional mathematical demography. New York: Wiley.

Schoen, R. (1988). Modeling multigroup populations. New York: Plenum.

Schoen, R. (2003). Dynamic populations with uniform natural increase across states. Mathematical Population Studies, 10, 195-210.

Schoen, R. and S.H. Jonsson. (2003). Modeling momentum in gradual demographic transitions. Demography, 40, 621-35.

Schoen, R. and Y.J. Kim. (1994a). Cyclically stable populations. Mathematical Population Studies, 4, 283-95.

Schoen, R. and Y.J. Kim. (1994b). Hyperstability. Paper presented at the Annual Meeting of the Population Association of America, Miami, May 5-7.

Schoen, R. and Y.J. Kim. (1996). Stabilization, birth waves, and the surge in the elderly. Mathematical Population Studies, 6, 35-53. 
Schoen: Intrinsically dynamic population models

Schoen, R. and Y.J. Kim. (1997). Exploring cyclic net reproduction. Mathematical Population Studies, 6, 277-90.

Schoen, R. and Y.J. Kim. (1998). Momentum under a gradual approach to zero growth. Population Studies, 52, 295-99.

Tuljapurkar, S. (1990). Population dynamics in variable environments (Vol. 85). New York: Springer.

Tuljapurkar, S.D. (1993). Entropy and convergence in dynamics and demography. Journal of Mathematical Biology, 31, 253-71.

Erratum: Varrious small mathematical corrections were made on pages $60,61,64$, and 65 on March 10, 2006. 


\section{Appendix}

1. In some cases, the series in equation (19) can be summed algebraically. For example, if

$$
\lambda_{1, t-\tau}=\frac{\lambda_{0}\{1+b \sin \omega[t-\tau]\}}{\{1+b \sin \omega[t-\tau-1]\}}
$$

Maple summation finds that $\left(\frac{1}{\mu_{M t}}\right)$ is of the form

$$
\begin{aligned}
& \left(\frac{1}{\mu_{M t}}\right)=1+C_{1 t}+C_{2 t} \sin \omega t+C_{3 t} \cos \omega t \\
& \text { where } \quad C_{1 t}=\frac{\lambda_{2}}{\left(\lambda_{0}-\lambda_{2}\right)(1+b \sin \omega t)} \\
& C_{2 t}=\frac{b \lambda_{2}\left(\lambda_{2}-\lambda_{0} \cos \omega\right)}{\left(\lambda_{2}^{2}-2 \lambda_{0} \lambda_{2} \cos \omega+\lambda_{0}^{2}\right)(1+b \sin \omega t)} \\
& \text { and } \quad C_{3 t}=\frac{-b \lambda_{0} \lambda_{2}(\sin \omega)}{\left(\lambda_{2}^{2}-2 \lambda_{0} \lambda_{2} \cos \omega+\lambda_{0}^{2}\right)(1+b \sin \omega t)} \text {. }
\end{aligned}
$$

2. The cubic characteristic equation of the 3 age group Leslie matrix given in equation (22) has the roots

$$
\begin{aligned}
& \lambda_{1}=\frac{\left[2 a+Z+\frac{\left(12 b+4 a^{2}\right)}{Z}\right]}{6} \\
& \lambda_{2}=\frac{\left[4 a-Z-\frac{\left(12 b+4 a^{2}\right)}{Z}\right]}{12}+\left(\frac{\sqrt{3} i}{12}\right)\left[Z-\frac{\left(12 b+4 a^{2}\right)}{Z}\right] \\
& \lambda_{3}=\frac{\left[4 a-Z-\frac{\left(12 b+4 a^{2}\right)}{Z}\right]}{12}-\left(\frac{\sqrt{3} i}{12}\right)\left[Z-\frac{\left(12 b+4 a^{2}\right)}{Z}\right] \text { where } \\
& Z=\left[36 a b+108 c+8 a^{3}+12\left[12 a^{3} c+81 c^{2}+54 a b c-3 a^{2} b^{2}-12 b^{3}\right]^{\frac{1}{2}}\right]^{\frac{1}{3}} .
\end{aligned}
$$


Schoen: Intrinsically dynamic population models 